\title{
Óleo de eucalipto e Pisolithus microcarpus no crescimento de bracatinga em solo contaminado por cobre
}

\author{
André L. Grolli ${ }^{5}$ \& Rudinei D. Marco ${ }^{6}$ \\ ${ }^{1}$ UFSM. Frederico Westphalen, RS. E-mail: adellai2@yahoo.com.br \\ ${ }^{2}$ UFSM. Frederico Westphalen, RS: E-mail: rodrigosilva@smail.ufsm.br (Autor correspondente) \\ ${ }^{3}$ UFSM. Frederico Westphalen, RS: E-mail: edison.perrando@ufsm.br \\ ${ }^{4}$ UFSM. Santa Maria, RS: E-mail: rodrigo@ufsm.br \\ ${ }^{5}$ UFSM. Frederico Westphalen, RS: Bolsista CAPES. E-mail: andregrolli1990@hotmail.com \\ ${ }^{6}$ UFSM. Frederico Westphalen, RS: Bolsista CAPES. E-mail: rudineidemarco@hotmail.com
}

Alex Dellai ${ }^{1}$, Rodrigo F. da Silva ${ }^{2}$, Edison R. Perrando ${ }^{3}$, Rodrigo J. S. Jacques ${ }^{4}$,

\section{Palavras-chave:}

ectomicorriza

arbórea nativa

metal pesado

Mimosa scabrella

\begin{abstract}
R E S U M O
As áreas de mineração de cobre podem apresentar teores elevados disponíveis deste nutriente no solo afetando o desenvolvimento vegetal. O objetivo do trabalho foi avaliar o efeito do óleo essencial de eucalipto na associação ectomicorrizica com o fungo Pisolithus microcarpus em mudas de bracatinga e seu comportamento em solo contaminado por cobre. O experimento foi conduzido em delineamento inteiramente casualizado em arranjo fatorial (4 x 5) sendo quatro formas de inoculação (testemunha, óleo, fungo e óleo + fungo) e cinco doses de cobre (nível

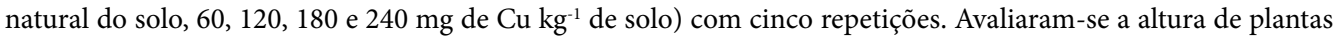
$(\mathrm{cm})$, diâmetro do colo $(\mathrm{mm})$, massa seca da parte aérea e radicular $(\mathrm{g})$, comprimento radicular $(\mathrm{cm})$, área superficial específica $\left(\mathrm{cm}^{2}\right)$, relação altura/diâmetro do colo e índice de qualidade de Dickson. Verificou-se que a utilização de $40 \mu \mathrm{L} \mathrm{L}^{-1}$ de óleo essencial de eucalipto aumenta a altura das mudas de bracatinga em solo com adição de $180 \mathrm{mg}$ de $\mathrm{Cu} \mathrm{kg}^{-1}$ de solo. Não há efeito da inoculação de Pisolithus microcarpus no crescimento das mudas de bracatinga em solo contaminado com cobre. As doses de cobre reduzem a massa seca da parte aérea e radicular das mudas.
\end{abstract}

Key words: ectomycorrhizal native tree heavy metal Mimosa scabrella

\section{Eucalyptus oil and Pisolithus microcarpus in the growth of bracatinga in soil contaminated by copper}

\begin{abstract}
A B S T R A C T
The copper mining areas may present elevated quantity available of this nutrient in the soil affecting the plant development. The objective of this research was to evaluate the effect of eucalyptus essential oil in the association of ectomycorrhizal with Pisolithus microcarpus fungus in bracatinga seedlings and its behavior in soil contaminated by copper. The experiment was conducted in a completely randomized delineation in a factorial arrangement (4 x 5), four types of inoculation (control, oil, fungus and oil + fungus) and five doses of copper (natural soil level, $60,120,180$ and $240 \mathrm{mg}$ of $\mathrm{Cu} \mathrm{kg}^{-1}$ soil) were used with five repetitions. Plant height $(\mathrm{cm})$, root collar diameter $(\mathrm{mm})$, dry mass of shoot and root $(\mathrm{g})$, root length $(\mathrm{cm})$, specific surface area $\left(\mathrm{cm}^{2}\right)$, and ratio of height/diameter of the collar and Dickson quality index were evaluated. It was verified that use of $40 \mu \mathrm{L} \mathrm{L}^{-1}$ of eucalyptus essential oil increases the bracatinga seedlings height in soil with addition $180 \mathrm{mg} \mathrm{of} \mathrm{Cu} \mathrm{kg}^{-1}$ soil. There is no effect of Pisolithus microcarpus inoculation in growth of bracatinga seedlings in soil contaminated with copper. The dose of copper reduces dry mass of shoot and root of seedlings.
\end{abstract}

\section{INTRODUÇÃ̃o}

O cobre, quando em altas concentrações no solo, pode diminuir a produção de plantas e ainda se acumular na cadeia alimentar (Santos et al., 2010). Além disto, pode oferecer riscos de contaminação de águas superficiais e subsuperficiais (Nowack et al., 2006). No Brasil, áreas contaminadas com cobre tem se tornado comum em vinhedos, decorrentes da aplicação de fungicidas à base de cobre (Nachtigall et al., 2007) e em áreas de mineração deste elemento (Regensburger et al., 2008). Diante disso, novas alternativas devem ser estudadas para diminuir a contaminação das áreas.

O uso de fungos micorrízicos pode ser uma alternativa para melhorar o desenvolvimento de plantas em áreas contaminadas. Esses fungos formam associações simbióticas com plantas vasculares aumentando a área explorada pelas raízes por meio de suas hifas no solo (Brundrett et al., 1996; Smith \& Read, 2008). Pesquisas têm indicado o efeito benéfico da associação micorrízica no crescimento vegetal (Silva et al., 2010; Magalhães et al., 2011) e demonstrado a eficiência desta associação na tolerância das plantas a metais pesados (Kabata-Pendias, 2010). 
De acordo com Smith \& Read (2008) os fungos ectomicorrízicos formam simbiose com espécies florestais específicas. Entretanto, metabólicos essenciais extraídos de espécies que formam associação com esses fungos podem contribuir para a ocorrência da simbiose em espécies nas quais, em condições naturais, não se tem detectado a ocorrência dessa associação (Steffen et al., 2012). Conforme Wenke et al. (2010) compostos exsudatos pelas raízes das plantas atuam como sinais bioquímicos essenciais. Referidos sinais estimulam a fase pré-simbiótica entre as raízes das plantas e os fungos (Steffen et al., 2011). Doran (1991) relatou que o óleo extraído de folhas de eucalipto contém uma mistura complexa envolvendo até 100 compostos orgânicos, que podem ter similaridade com compostos exsudatos pelas raízes das plantas atuando como sinal bioquímico para a ocorrência da associação simbiótica, caso em que o óleo essencial de eucalipto poderá ser uma alternativa para estimular a ocorrência de associação ectomicorrízica em espécies nativas que apresentem dificuldade em se associar com fungos ectomicorrizicos.

As espécies florestais acumulam metais pesados nas raízes e no caule, sendo importantes para recuperar ambientes contaminados por metais (Magalhães et al., 2011). A bracatinga (Mimosa scabrella Benth), por exemplo, é uma Fabaceae adequada para a recuperação de áreas degradadas visto que deposita quantidades expressivas de material orgânico rico em nitrogênio no solo (Carpanezzi et al., 1988). Regensburger et al. (2008) verificaram 92\% de índice de sobrevivência da bracatinga em áreas degradadas pela mineração de argila; entretanto, não se tem evidenciado trabalhos sobre o comportamento de fabaceas, como a bracatinga, em solo contaminado com cobre. Deste modo, a elaboração de estudos que contemplem o desenvolvimento desta espécie florestal, em solo contaminado por cobre, poderá contribuir para o conhecimento sobre arbóreas nativas do Brasil em solo contaminado.

O objetivo do trabalho foi avaliar o efeito do óleo essencial de eucalipto na associação ectomicorrizica com o fungo Pisolithus microcarpus em bracatinga e seu comportamento em solo contaminado por cobre.

\section{Material e Métodos}

O experimento foi conduzido em casa de vegetação. A espécie utilizada foi a bracatinga (Mimosa scabrella (Benth)) cujas sementes foram fornecidas pela Estação de Pesquisas Florestais de Santa Maria - FEPAGRO FLORESTAS. As sementes tiveram a superfície desinfetada com hipoclorito de sódio $2 \%$ durante 10 min e álcool $70 \%$ por mais 12 min e lavadas em água esterilizada. Para a superação de dormência tegumentar das sementes foi realizada escarificação mecânica do lado oposto ao embrião, com lixa Norton 80 .

O substrato utilizado foi um Latossolo Vermelho, com a seguinte análise química e física: $\mathrm{pH}_{\text {agua }}: 4,9, \mathrm{Ca}+\mathrm{mg}: 5,4 \mathrm{cmol}_{\mathrm{c}}$ $\mathrm{kg}^{-1}, \mathrm{Al}: 4,3 \mathrm{cmol}_{\mathrm{c}} \mathrm{kg}^{-1}, \mathrm{H}+\mathrm{Al}: 6,6 \mathrm{cmol}_{\mathrm{c}} \mathrm{kg}^{-1}, \mathrm{P}: 6,6 \mathrm{mg} \mathrm{dm}^{-3}, \mathrm{~K}^{\mathrm{C}}$ $111,0 \mathrm{mg} \mathrm{dm}^{-3}, \mathrm{Zn}: 1,6 \mathrm{mg} \mathrm{dm}^{-3}, \mathrm{Cu}: 15,1 \mathrm{mg} \mathrm{dm}^{-3}, \mathrm{MO}: 2,4 \%$, Argila: $81 \%$. O solo foi esterilizado três vezes em autoclave na temperatura de $121{ }^{\circ} \mathrm{C}$, durante $60 \mathrm{mim}$.
A semeadura foi realizada em tubetes de polipropileno com capacidade de $125 \mathrm{~cm}^{3}$, utilizando-se três sementes por tubete sendo cada tubete considerado uma unidade experimental (UE). Quando as mudas apresentavam um par de folhas definitivas, procedeu-se a um desbaste, permanecendo apenas uma muda por tubete, considerando-se o aspecto de fitossanidade e vigor.

O isolado ectomicorrízico utilizado foi o UFSC-Pt116 (Pisolhitus microcarpus) pertencente ao banco de fungos da Universidade Federal de Santa Catarina. O fungo foi multiplicado em placas de Petri contendo $20 \mathrm{~mL}$ meio MNM (Melin-Norkrans Modificado) Marx (1969) mantido em BOD a $28^{\circ} \mathrm{C}$ com ausência de luz. O conteúdo da placa de Petri (Micélio fúngico e meio $\mathrm{MNM}$ ) foi fragmentado em liquidificador com $200 \mathrm{~mL}$ de água esterilizada durante $10 \mathrm{~s}$; posteriormente, foram aplicados $2 \mathrm{~mL}$ dessa suspensão miceliana nas UE cujo procedimento foi realizado cinco veze, durante o período de condução do experimento, em intervalos de 15 dias, partindose da semeadura; nos tratamentos sem a inoculação de fungos foi aplicado apenas o meio MNM.

O óleo essencial utilizado foi de Eucalyptus grandis obtido através da metodologia descrita por Vitti \& Britto (2003). A concentração do óleo essencial utilizado no experimento seguiu as indicadas por Steffen et al. $(2011 ; 2012)$ que avaliaram doses de óleo essencial de eucalipto para estimular a micorrização de mudas de Eucalyptus grandis e de sibipiruna (Caesalpinia peltophoroides) sendo utilizada, nesses experimentos, a concentração de $40 \mu \mathrm{L} \mathrm{L}^{-1}$. Foram realizadas quatro aplicações contendo $2 \mathrm{~mL}$ da solução de óleo essencial, a primeira 12 dias após a emergência das plântulas e as demais em intervalos de 15 dias. As adubações de cobertura seguiram as recomendações propostas por (Gonçalves \& Benedetti, 2005).

O delineamento experimental foi inteiramente casualizado em arranjo fatorial (4 x 5) Qualitativo em A e Quantitativo em $\mathrm{D}$, sendo fator A quatro formas de inoculação: testemunha (sem fungo e óleo); óleo (adição de óleo); fungo (adição do fungo) e óleo + fungo (adição de óleo e fungo) e fator $\mathrm{D}$ cinco doses de cobre (0 (teor natural do solo), 60, 120, 180 e $240 \mathrm{mg}$ de $\mathrm{Cu}$ $\mathrm{kg}^{-1}$ de solo) com cinco repetições. A avaliação foi aos 180 dias após a semeadura.

Os parâmetros avaliados foram altura de planta (AP) em $\mathrm{cm}$, diâmetro do colo (DC) em $\mathrm{mm}$, massa seca da parte aérea (MSPA) e radicular (MSPR) em g, comprimento da raiz principal (CRP) em cm, área superficial específica radicular (ASE) $\mathrm{em} \mathrm{cm}^{2} \mathrm{e}$ porcentagem de colonização radicular (CM\%). A altura de plantas foi obtida com régua graduada de $30 \mathrm{~cm}$ e pela distância do colo até as últimas axilas foliares. $\mathrm{O}$ diâmetro do colo foi mensurado com o auxílio de um paquímetro digital. Para a avaliação da MSPA e MSPR a parte aérea da radicular foi separada e secada em estufa em temperatura de $65^{\circ} \mathrm{C}$, até apresentar peso constante; em seguida, ambas as partes foram pesadas em balança analítica.

Para obtenção da porcentagem de colonização radicular as raízes foram submetidas ao procedimento de clareamento e coloração, que constaram em deixar uma amostra de $0,1 \mathrm{~g}$ de raízes imersas em solução de $\mathrm{KOH} 10 \%$, a $80{ }^{\circ} \mathrm{C}$ durante $1 \mathrm{~h}$ e $30 \mathrm{~min}$, após foram levadas com água e, posteriormente, as raízes foram colocadas em $\mathrm{HCl} 0,1 \mathrm{M}$ durante 2 min lavando-as 
novamente com água e colocadas em Trypam Blue (corante) a $80{ }^{\circ} \mathrm{C}$ por $30 \mathrm{~min}$ (Brundrett et al., 1996). A quantificação da porcentagem de colonização radicular foi obtida pelo método da placa quadriculada (Brundret et al., 1996).

Com base nos parâmetros morfológicos avaliados foram calculados os índices de qualidade de mudas: relação entre altura da parte aérea e diâmetro do coleto (H/DC) e o Índice de Qualidade de Dickson (IQD), Dickson et al. (1960): IQD = (MSPA + MSPR) / [AP/DC]+[MSPA/MSPR].

Para atender aos pressupostos da análise de variância, os valores médios do comprimento radicular, massa seca da parte aérea, massa seca da parte radicular e índice de qualidade de Dickson foram transformados pela equação $\left(\mathrm{x}^{0,5}\right)$. Os resultados foram submetidos à análise de variância e quando apresentaram interação significativa entre $\mathrm{A} x \mathrm{D}$ foram submetidos à análise de regressão e ao teste de comparação de média de Tukey, com nível de 0,05 de probabilidade de erro, pelo programa Sisvar (Ferreira, 2011). Para os parâmetros sem interação significativa foram desdobrados os efeitos simples com nível de 0,05 de probabilidade de erro.

\section{Resultados e Discussão}

O porcentual de colonização radicular apresentou interação significativa entre as doses (Fator D) e tratamentos (Fator A). A formação de micorriza ocorreu nas doses 0,60 e $120 \mathrm{mg} \mathrm{kg}^{-1}$, nos tratamentos fungo e óleo + fungo, sendo que na dose $60 \mathrm{mg} \mathrm{kg}^{-1}$ o maior porcentual de colonização radicular foi no tratamento óleo + fungo (Figura 1). Steffen et al. (2011) verificaram que o óleo essencial de eucalipto estimulou a porcentagem de colonização radicular em mudas de Eucalyptus grandis, atribuindo ao óleo essencial estar atuando de forma efetiva no quimiotropismo entre hifas do fungo e células radiculares. Entretanto, a porcentagem de colonização ectomicorrízica encontrada neste trabalho foi inferior às encontradas em mudas de eucalipto (Steffen et al., 2011) e sibipiruna (Steffen et al., 2012) respectivamente de 40 e $20 \%$, com aplicação de $40 \mu \mathrm{L} \mathrm{L}^{-1}$ de óleo essencial de eucalipto. As doses de cobre a partir de $180 \mathrm{mg} \mathrm{kg}^{-1}$ inibiram a formação da ectomicorriza nas mudas de bracatinga.
Os resultados também evidenciaram interação significativa entre doses (Fator D) e tratamentos (Fator A) para as variáveis: altura, diâmetro, relação H/DC e índice de qualidade de Dickson (Tabela 1, Figura 2). Verifica-se que as doses de cobre causaram efeito negativo para a altura das mudas de

Tabela 1. Altura, diâmeto do colo, relação altura diâmetro do colo (H/DC) e índice de qualidade de Dickson (IQD) de mudas de bracatinga submetidas aos tratamentos: testemunha, óleo, fungo e óleo + fungo em solo contaminado com doses de cobre

\begin{tabular}{|c|c|c|c|c|}
\hline $\begin{array}{c}\text { Dose de Cu } \\
\mathrm{mg} \mathrm{kg}^{-1}\end{array}$ & Testemunha & Óleo & Fungo & Óleo + Fungo \\
\hline & \multicolumn{4}{|c|}{ Altura (cm) } \\
\hline 0 & $9,5 a A^{*}$ & $8,9 \mathrm{abA}$ & $8,70 \mathrm{abA}$ & $9,2 \mathrm{aA}$ \\
\hline 60 & $8,7 \mathrm{abA}$ & $9,0 \mathrm{abA}$ & $9,40 \mathrm{aA}$ & $9,5 \mathrm{aA}$ \\
\hline 120 & $8,4 \mathrm{abAB}$ & $8,0 \mathrm{bAB}$ & $7,68 \mathrm{bB}$ & $9,0 \mathrm{aA}$ \\
\hline 180 & 7,7 bB & $9,4 \mathrm{aA}$ & $8,44 a b A B$ & $8,6 \mathrm{aAB}$ \\
\hline 240 & $8,2 \mathrm{bA}$ & $8,9 a b A$ & $8,32 \mathrm{abA}$ & $8,8 \mathrm{aA}$ \\
\hline \multirow[t]{2}{*}{ CV\% } & \multicolumn{4}{|c|}{7,57} \\
\hline & \multicolumn{4}{|c|}{ Diâmetro do Colo (mm) } \\
\hline 0 & $1,02 \mathrm{aAB}$ & $0,92 \mathrm{aB}$ & $1,19 \mathrm{aA}$ & $1,07 \mathrm{aAB}$ \\
\hline 60 & $0,95 \mathrm{aA}$ & $0,92 \mathrm{aA}$ & $0,89 \mathrm{bA}$ & $0,97 \mathrm{abA}$ \\
\hline 120 & $0,93 \mathrm{aA}$ & $0,83 \mathrm{aA}$ & $0,82 \mathrm{bA}$ & $0,84 \mathrm{abA}$ \\
\hline 180 & $0,79 a A B$ & $0,95 \mathrm{aA}$ & $0,72 \mathrm{bB}$ & $0,83 \mathrm{bAB}$ \\
\hline 240 & 0,85 aA & $0,92 \mathrm{aA}$ & $0,82 \mathrm{bA}$ & $0,78 \mathrm{bA}$ \\
\hline \multirow[t]{2}{*}{ CV\% } & \multicolumn{4}{|c|}{14,79} \\
\hline & \multicolumn{4}{|c|}{$\mathrm{H} / \mathrm{DC}$} \\
\hline 0 & $9,6 \mathrm{aAB}$ & $9,7 \mathrm{aA}$ & $7,7 \mathrm{cB}$ & $8,9 \mathrm{bAB}$ \\
\hline 60 & $9,3 \mathrm{aA}$ & $10,0 \mathrm{aA}$ & $10,5 a b A$ & $10,0 a b A$ \\
\hline 120 & $9,0 \mathrm{aA}$ & $9,7 \mathrm{aA}$ & $9,3 \mathrm{bcA}$ & $10,7 \mathrm{abA}$ \\
\hline 180 & $9,7 \mathrm{aB}$ & $9,8 \mathrm{aAB}$ & $11,7 \mathrm{aA}$ & $10,3 \mathrm{abAB}$ \\
\hline 240 & $9,6 \mathrm{aA}$ & $9,8 \mathrm{aA}$ & $10,2 a b A$ & $11,4 \mathrm{aA}$ \\
\hline \multirow[t]{2}{*}{$\mathrm{CV} \%$} & \multicolumn{4}{|c|}{12,03} \\
\hline & \multicolumn{4}{|c|}{ IQD } \\
\hline 0 & $0,016 \mathrm{aA}$ & $0,015 \mathrm{abA}$ & $0,022 \mathrm{aA}$ & $0,017 \mathrm{abA}$ \\
\hline 60 & $0,018 \mathrm{aA}$ & $0,015 \mathrm{abA}$ & $0,013 \mathrm{abA}$ & $0,022 \mathrm{aA}$ \\
\hline 120 & $0,013 \mathrm{aA}$ & $0,010 \mathrm{bA}$ & $0,011 \mathrm{abA}$ & $0,010 \mathrm{abA}$ \\
\hline 180 & $0,010 \mathrm{aB}$ & $0,024 \mathrm{aA}$ & 0,006 bB & $0,009 a b B$ \\
\hline 240 & $0,009 a A$ & $0,012 a b A$ & $0,011 \mathrm{abA}$ & 0,007 bA \\
\hline CV\% & \multicolumn{4}{|c|}{24,45} \\
\hline
\end{tabular}

* Médias seguidas de mesma letra minúscula na coluna e maiúscula na linha não diferiram entre si pelo teste de Tukey 0,05 de probabilidade de erro

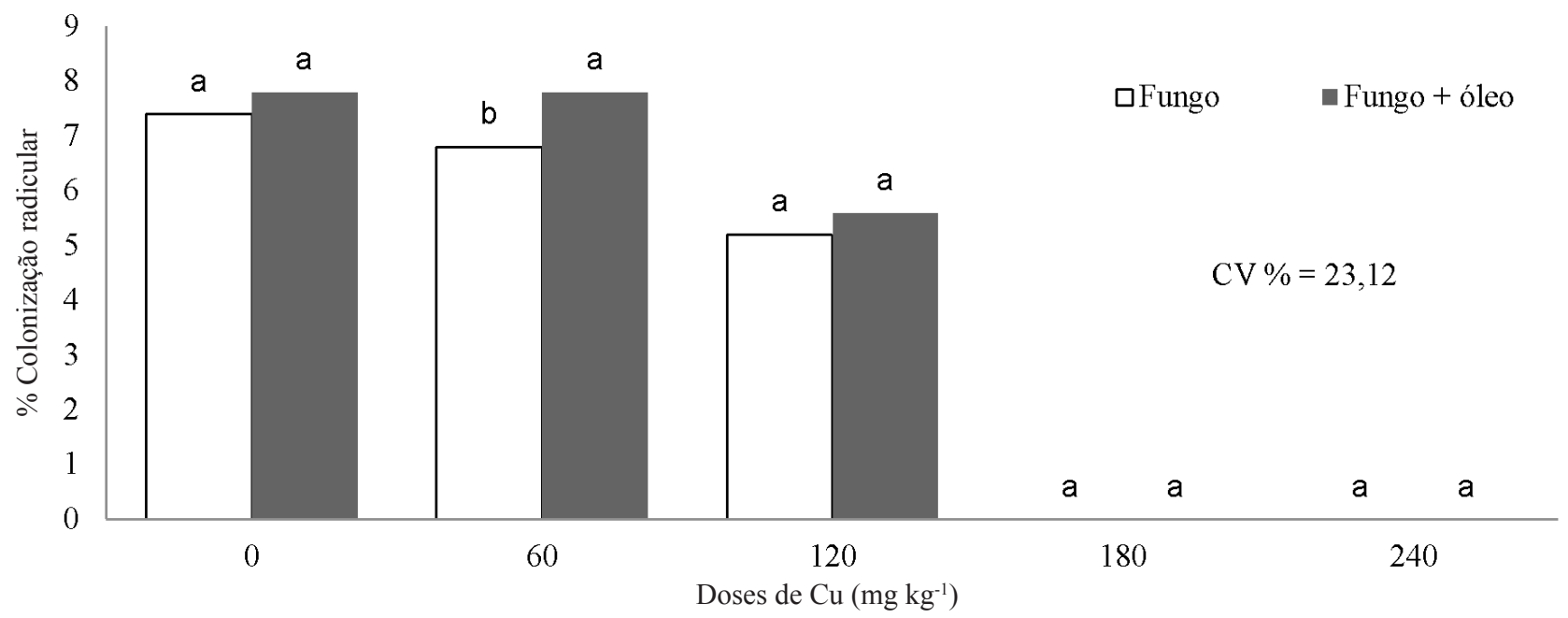

Figura 1. Porcentual de colonização radicular de mudas de bracatinga obtidas nos tratamentos fungo e óleo + fungo, submetidas às doses de $0,60,120,180$ e $240 \mathrm{mg}$ de $\mathrm{Cu} \mathrm{kg}^{-1}$ de solo 
- Testemunha - Óleo essencial

A.

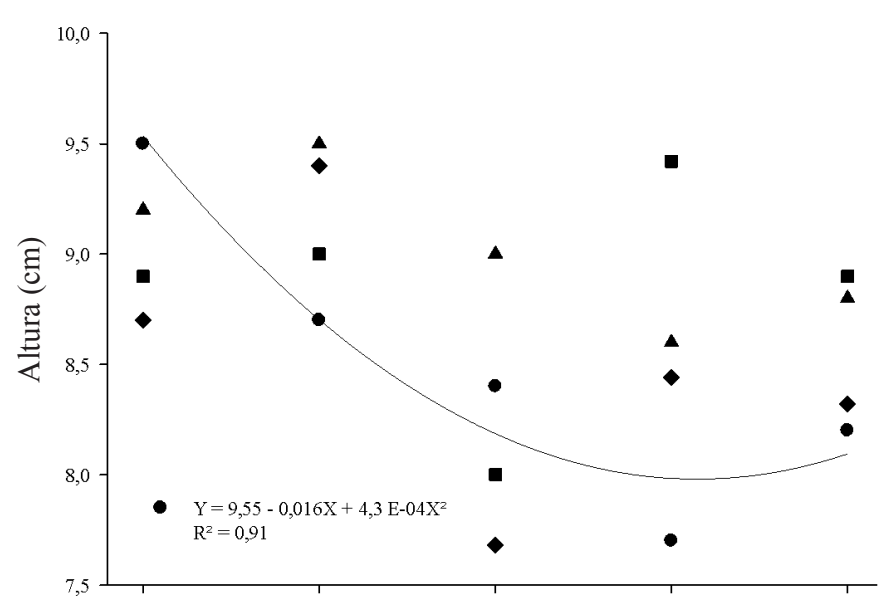

C.

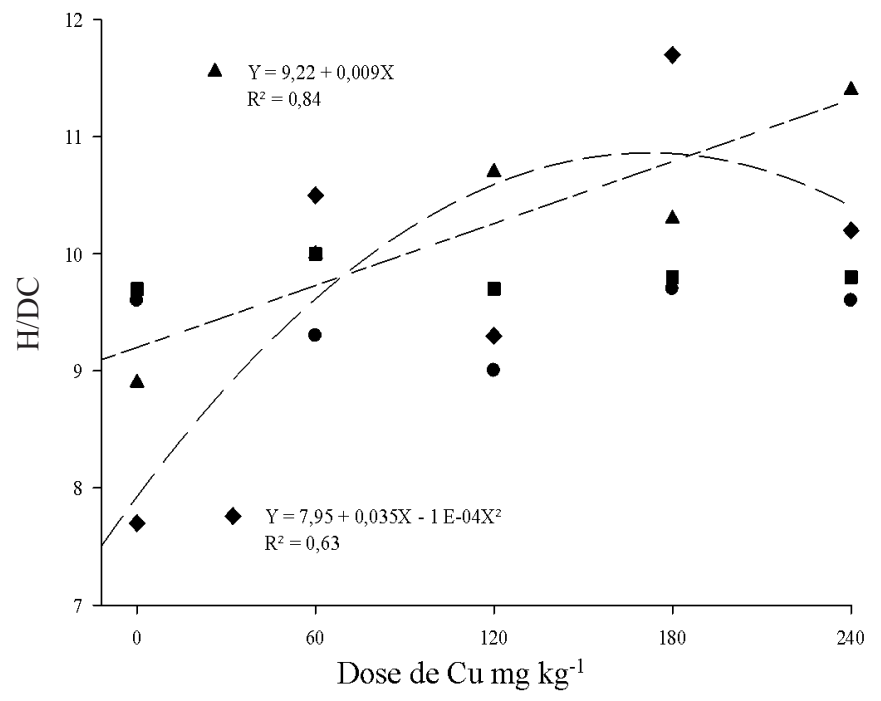

- fungo $\Delta$ Óleo + fungo *

B.

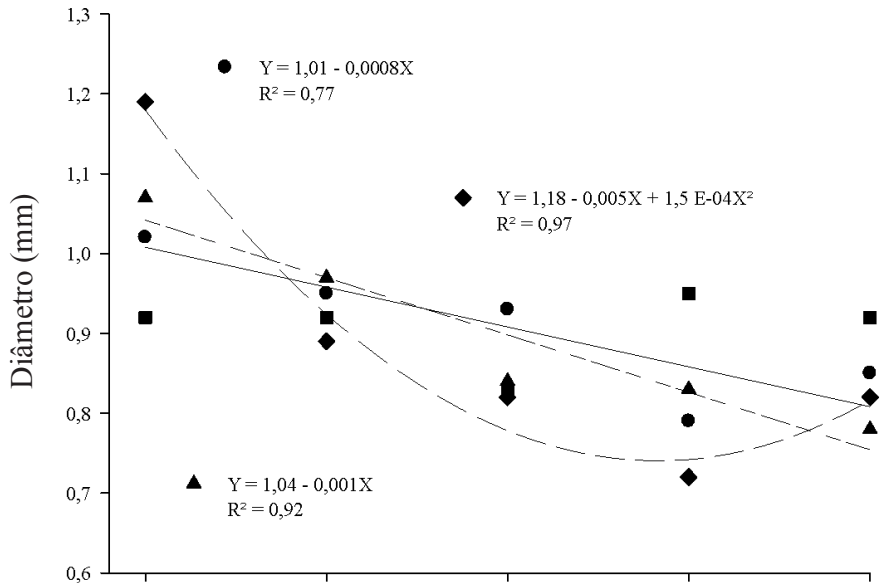

D.

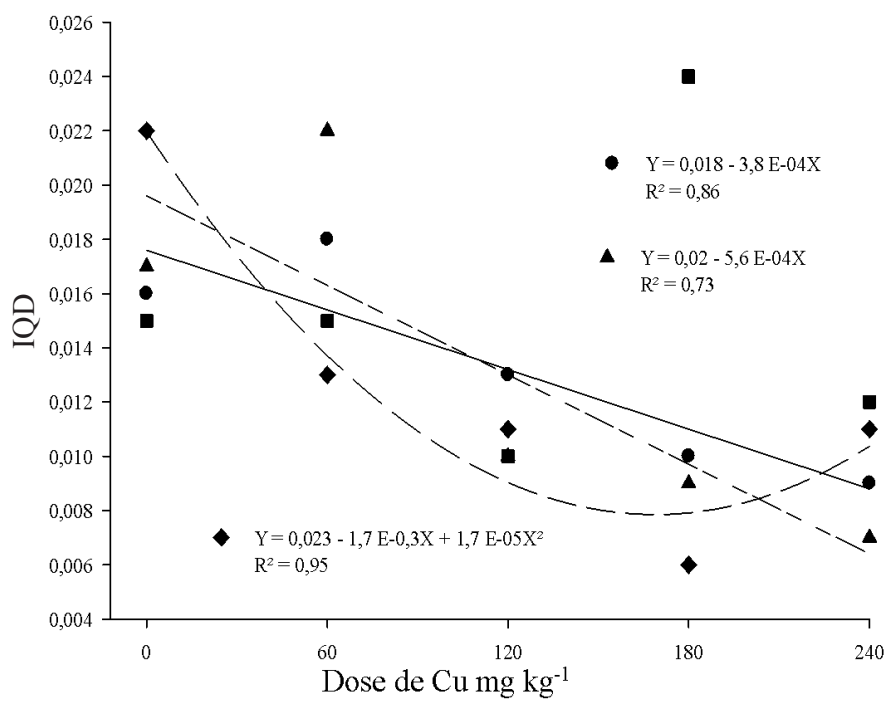

*Somente equações de regressão com $\mathrm{R}^{2}>0,60$ foram ajustadas e apresentadas

Figura 2. Equações de regressão para altura (A), diâmetro do colo (B), relação altura/diâmetro do colo (H/DC) (C) e índice de qualidade de Dickson (IQD) (D) das mudas de bracatinga submetidas aos tratamentos: testemunha, óleo, fungo e óleo + fungo, em solo contaminado com doses de cobre

bracatinga com efeito significativo para testemunha (Figura 2) e redução linear para o tratamento óleo + fungo $(Y=9,41$ - 0,003x; $\left.R^{2}=0,56\right)$ porém a utilização de óleo possibilitou maior altura na dose de $180 \mathrm{mg} \mathrm{kg}^{-1}$ de cobre (Tabela 1). O uso de óleos essenciais sobre as plantas ativa mecanismos de defesa, ou reguladores de crescimento, como fitoalexinas e citocininas (Bonaldo et al., 2007). A produção de citocininas pode resultar em maior desenvolvimento vegetal (Taiz \& Zeiger, 2012). Assim, a utilização de óleo essencial de eucalipto parece beneficiar a altura de mudas de bracatinga em solo com elevada concentração de cobre.

O diâmetro do colo foi reduzido significativamente nos tratamentos fungo e óleo + fungo com o incremento das doses de cobre aplicadas ao solo, diferindo do tratamento óleo somente na dose de $180 \mathrm{mg} \mathrm{kg}^{-1}$ (Tabela 1; Figura 2B). Porém na dose zero os valores observados para o diâmetro do colo nos tratamentos fungo e óleo + fungo foram significativamente superiores aos demais, em 16,6 e 4,9\%, respectivamente. Mello et al. (2009) também verificaram maior diâmetro do colo em mudas micorrizadas, no estabelecimento Eucalyptus grandis em solo arenoso. Referidos resultados indicam que a inoculação do fungo UFSC-Pt116 pode promover aumentos no diâmetro do colo das mudas de bracatinga em solo não contaminado por cobre. Entretanto, o uso do óleo essencial, quando comparado ao tratamento fungo, possibilita crescimento em diâmetro, mesmo com a elevação das doses de cobre no solo. De acordo com Steffen et al. (2010) o óleo essencial pode ser um indutor biológico de crescimento vegetal, o que poderia explicar o crescimento em diâmetro neste estudo.

Observou-se que os tratamentos fungo e óleo + fungo possibilitaram aumento na relação H/DC com a adição das doses de cobre ao solo (Figura 2C) e somente o tratamento fungo difeririu significativamente da testemunha na dose 180 $\mathrm{mg} \mathrm{kg} \mathrm{kg}^{-1}$ (Tabela 1). Entretanto, os valores observados em todos 
os tratamentos estão acima da faixa adequada para o transplante, que varia de 5,4 a 8,1 (Carneiro, 1995) indicando que a inoculação de Pisolhitus microcarpus embora contribua para o diâmetro do colo de bracatinga (Tabela 1) quando submetido a elevada dose de cobre, poderá induzir ao estiolamento das mudas de bracatinga.

O índice de qualidade de Dickson foi reduzido linearmente nos tratamentos testemunha e óleo + fungo e de forma quadrática negativa no tratamento fungo, com as doses de cobre (Figura 2). Contudo, este índice foi maior no tratamento óleo, em relação aos demais tratamentos qualitativos, na dose de $180 \mathrm{mg} \mathrm{kg}^{-1}$ (Tabela 1). Os óleos essenciais podem atuar como indutores biológicos no crescimento vegetal contribuindo para a melhoria na qualidade de mudas (Steffen et al., 2011). Para o cálculo do IQD são considerados atributos morfológicos os quais expressam a robustez da muda e, neste caso, é possível que os valores baixos observados no trabalho sejam devidos ao maior crescimento em altura ou à menor produção de massa seca radicular das mudas, cujos resultados indicam que o uso de óleo essencial de eucalipto pode contribuir para o IQD das mudas de bracatinga em doses elevadas de cobre.

Para os parâmetros sem interação significativa verificou-se que a massa seca da parte aérea e radicular, comprimento e área superficial específica, foram afetados significativamente com as doses de cobre (Figura 3). A massa seca da parte aérea foi reduzida linearmente com as doses de cobre, sendo $58 \%$ menor com 240 mg de cobre kg-1 de solo e DL50 em 230,5 mg $\mathrm{kg}^{-1}$ de cobre (Figura 3A). De acordo com Grassi Filho (2005) plantas submetidas a doses elevadas de cobre podem apresentar desfolhamento precoce além de redução no crescimento da parte aérea. Este resultado corrobora com os de Silva et al. (2011) que também encontraram redução na massa seca da parte aérea de arbóreas nativas como a canafístula (Peltophorum dubium) em doses elevadas de cobre no solo.

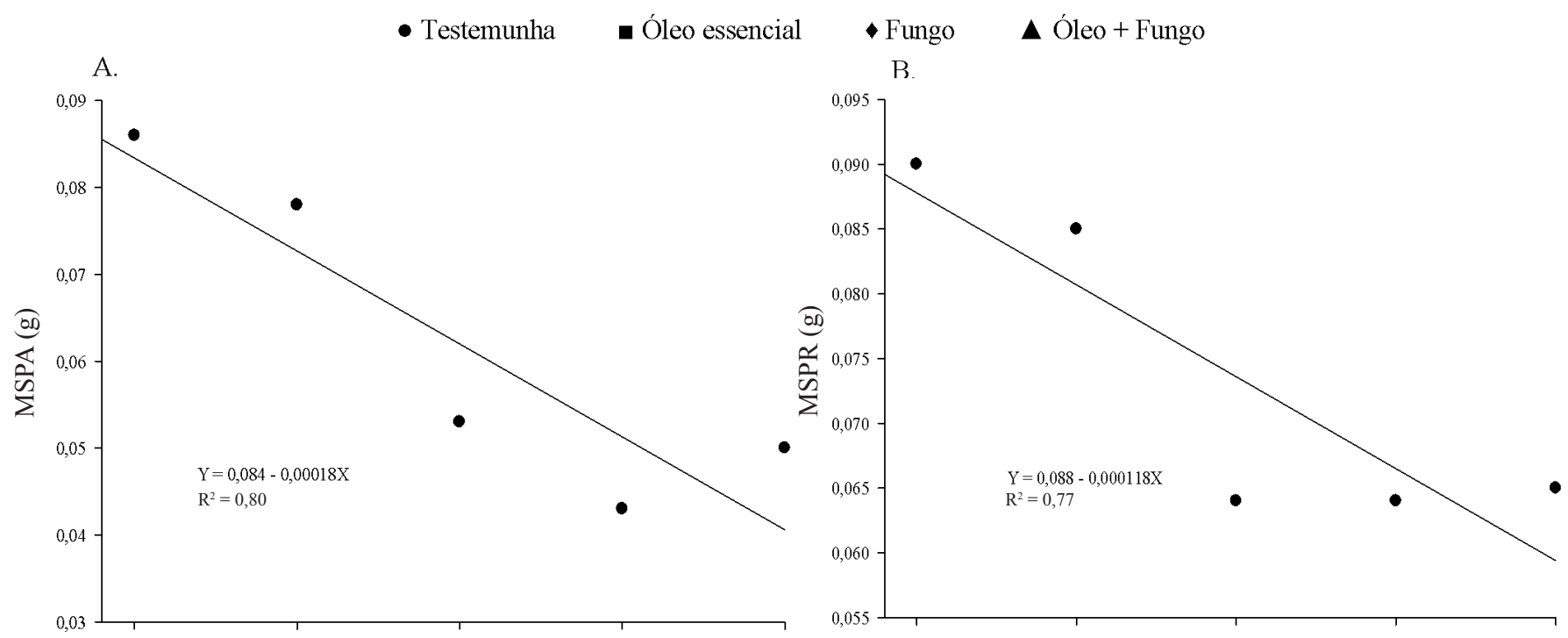

C.
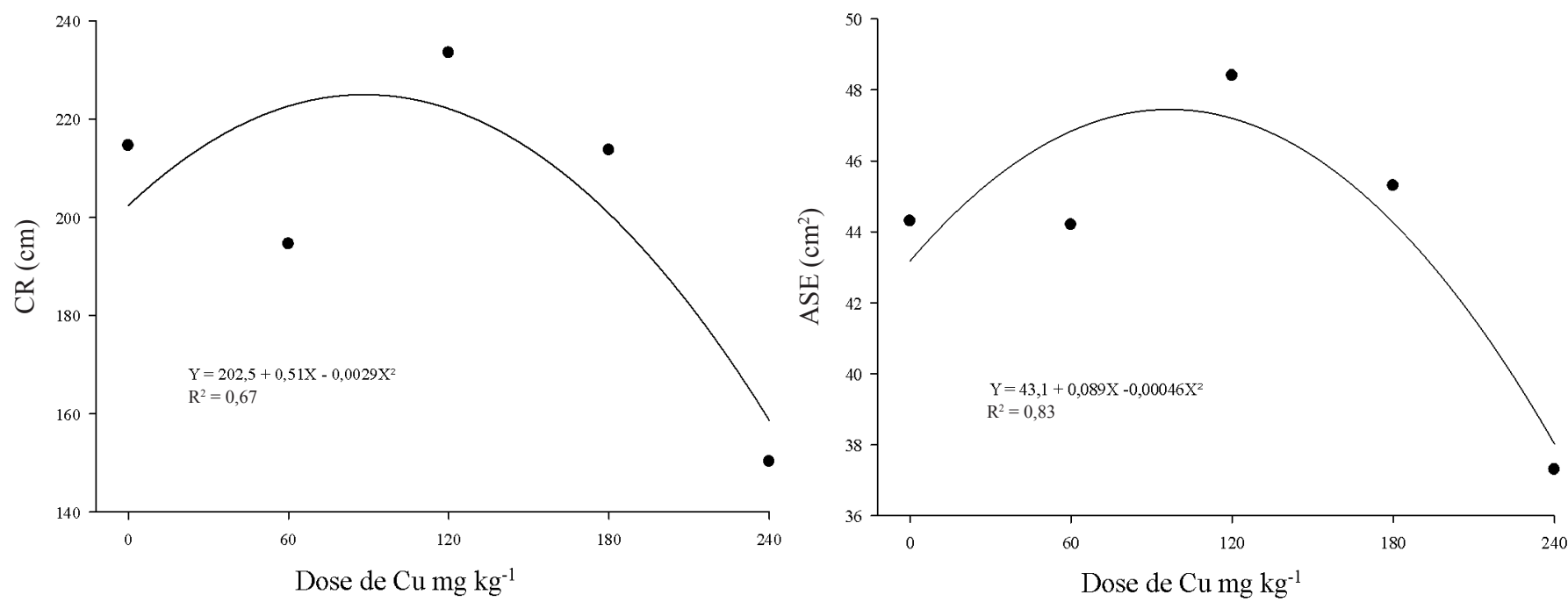

Figura 3. Massa seca da parte aérea (MSPA) (A), massa seca da parte radicular (MSPR) (B), comprimento radicular (CR) (C), Área superficial específica (ASE) (D) das mudas de bracatinga submetidas aos tratamentos: testemunha, óleo, fungo e fungo + óleo, em solo contaminado com doses de cobre 
A massa seca da parte radicular também foi reduzida linearmente sendo $72 \%$ menor na dose $240 \mathrm{mg} \mathrm{kg}^{-1}$, quando comparada com a das mudas produzidas no tratamento sem aplicação de cobre (Figura 3B). Resultados semelhantes foram observados por Silva et al. (2012) para mudas de dedaleiro (Lafoensia pacari) produzidas em solo contaminado com cobre. Entretanto, embora a produção da massa seca da parte radicular das mudas de bracatinga tenha sido reduzida pela aplicação das doses de cobre, a DL50 estimada foi de $364,4 \mathrm{mg} \mathrm{kg}^{-1}$ de cobre (Figura 3B) indicando que o cobre afeta, primeiro, a parte aérea das mudas de bracatinga.

O comprimento radicular (Figura 3C) e a área superficial específica (Figura 3D) das raízes da bracatinga apresentaram ponto de máxima na dose de 88 e $97 \mathrm{mg} \mathrm{kg}^{-1}$, respectivamente. Silva et al. (2011) também observaram que a partir de determinada dose a contaminação com cobre reduziu o comprimento radicular em mudas de aroeira vermelha (Schinus terebinthifolius). Conforme Bellion et al. (2006) o cobre interfere, quando absorvido no sistema radicular, na expansão celular, atuando diretamente na redução das raízes (Panou-Filotheu et al., 2001). Portanto, o cobre em altas concentrações no solo pode causar efeitos negativos no sistema radicular de mudas de bracatinga.

Não se verificou efeito significativo dos tratamentos qualitativos nos valores de massa seca da parte aérea, massa seca da parte radicular, comprimento radicular e área superficial específica radicular. Trabalhos de pesquisa têm demonstrado o efeito positivo da adição do óleo essencial de eucalipto em espécies florestais (Steffen et al., 2012), bem como da inoculação com o fungo ectomicorrízico Pisolhitus microcarpus na massa seca da parte aérea de mudas de Eucalyptus grandis (Souza et al., 2012). Entretanto, mesmo ocorrendo colonização, ainda é possível que a simbiose não seja funcional ou que o fungo atue como parasita da planta em situação de estresse (Smith \& Read, 2008). Desta forma, embora tenha ocorrido a formação de ectomicorriza nas menores doses de cobre (Figura 1), não foi possível evidenciar efeito dos tratamentos qualitativos na massa seca da parte aérea e radicular, comprimento e área superficial específica radicular das mudas de bracatinga.

\section{Conclusões}

1. A utilização de $40 \mu \mathrm{L} \mathrm{L}^{-1}$ de óleo essencial de eucalipto aumenta a altura das mudas de bracatinga em solo argiloso contaminado com adição de $180 \mathrm{mg}$ de cobre $\mathrm{kg}^{-1}$ de solo.

2. Não há efeito da inoculação de Pisolithus microcarpus no crescimento das mudas de bracatinga em solo contaminado com cobre.

3. As doses de cobre reduzem a massa seca da parte aérea e radicular das mudas de bracatinga.

\section{Agradecimentos}

À CAPES, pela concessão da bolsa de Mestrado e ao CNPQ, pela bolsa de iniciação científica.

\section{Literatura Citada}

Bellion, M; Courbot, M.; Jacob, C.; Blaudez, D.; Chalot, M. Extracellular and celullar mechanisms sustaining metal tolerance in ectomycorrizal fungi. FEMS Microbiology Letters, v.254, p.173-181, 2006.

Bonaldo, S. M.; Schwan-Estrada, K. R. F.; Stangarlin, J. R.; Cruz, M. E. S.; Fiori-Tutida, A. C. G. Contribuição ao estudo das atividades antifúngica e elicitora de fitoalexinas em sorgo e soja por eucalipto (Eucalyptus citriodora). Summa Phytopathologica, v.33, p.383-387, 2007.

Brundrett, M.; Bougher, N.; Dell, B. Working with mycorrhizal in forestry and agriculture. Canberra: ACIAR, 1996. 400p.

Carneiro, J. G. A. Produção e controle de qualidade de mudas florestais. Curitiba: UFPR/FUPEF, 1995. 451p.

Carpanezzi, A.; Laurent, J. M. E.; Carvalho, P. E. R.; Pegoraro, A.; Baggio, A. J.; Zanon, A.; Oliveira, E. B.; Lede, E. T.; Rotta, E.; Sturion, J. A.; Pereira, J. C. D.; Graça, L. R.; Rauen, M. J.; Carpanezzi, O. T. B.; Oliveira, Y. M. M. Manual técnico da bracatinga (Mimosa scabrella Benth), Colombo: Embrapa CNPF. 1988. 70p. Documentos, 20

Dickson, A.; Leaf, A. L.; Hosner, J. F. Quality appraisal of white spruce and white pine seedling stock in nurseries. Forestry Chronicle, v.36, p.10-13, 1960.

Doran, J. C. Commercial sources, uses, formation, and biology. In: Boland, D. J.; Brophy, J. J.; House, A. P. N. Eucalyptus leaf oils: Use, chemistry, distillation and marketing. Melbourne: Inkata, 1991. p.11-28.

Ferreira, D. F. Sisvar: A computer statistical analysis system. Ciência e Agrotecnologia, v.35, p.1039-1042, 2011.

Gonçalves, J. L. M.; Benedetti, V. Nutrição e fertilização florestal. Piracicaba: Filipel, 2005. 427p.

Grassi Filho, H. Cobre na planta. 2005. Universidade Estadual Paulista. Faculdade de Ciências Agronômicas. <http://www.ciencialivre.pro.br / media/5d14138ca192e610ffff81e2ffffd523.pdf $>.15$ Mai 2013.

Kabata-Pendias, A. Trace elements in soils and plants. 4.ed. New York: Boca Raton, 2010.548p.

Magalhães, M. O. L.; Amaral Sobrinho, M. B. do; Santos, F. S. dos; Mazur, N. Potencial de duas espécies de eucalipto na fitoestabilização de solo contaminado com zinco. Revista Ciência Agronômica, v.42, p.805-812, 2011.

Marx, D. H. The influence of ectotrophic mycorrhizal fungi on the resistance of pine roots to pathogenic infections. I. Antagonism of mycorrhizal fungi to root pathogenic fungi and soil bacteria. Phytopathologist, v.59, p.153-163, 1969.

Mello, A. H.; Antoniolli, Z. I.; Kaminski, J.; Souza, E. L.; Schirmer, J. K.; Machado, R. G.; Lupatini, M.; Moro Júnior, C. Estabelecimento a campo de mudas de Eucalyptus grandis micorrizadas com Pisolithus microcarpus (UFSC-Pt116) em solo arenoso. Ciência Florestal, v.19, p.149-155, 2009.

Nachtigall, G. R.; Nogueirol, C. R.; Alleoni, L. R. F.; Cambri, M. A. Copper concentration of vineyard soils as a function of $\mathrm{pH}$ variation and addition of poultry litter. Brazilian Archives of Biology and Technology, v.50, p.941-948, 2007. 
Nowack, B.; Schulin, R.; Robinson, B. H. Critical assessment of chelant-enhanced metal phytoextraction. Environmental Science and Technology, v.40, p.5525-5532, 2006.

Panou-Filotheu, H. Bosabalidis, A. M.; Karataglis, S. Effects of copper toxicity on leaves of oregano (Origanum vulgare subsp. Hirtum). Annals of Botany, v.88, p.207-214, 2001.

Regensburger, B.; Comin, J. J.; Aumond, J. J. Integração de técnicas de solo, plantas e animais para recuperar áreas degradadas. Ciência Rural, v.38, p.1773-1776, 2008.

Santos, G. C. G.; Rodella, A. A.; Abreu, C. A.; Coscione, A. R. Vegetable species for phytoextraction of boron, copper, lead, manganese and zinc from contaminated soil. Scientia Agricola, v.67, p.713-719, 2010.

Silva, R. F.; Antoniolli, Z. I.; Lupatini, M.; Trindade, L. L.; Silva, A. S. Tolerância de mudas de canafístula (Peltophorum dubium (spreng.) taub.) inoculada com Pisolithus microcarpus a solo com excesso de cobre. Ciência Florestal, v.20, p.147-156, 2010.

Silva, R. F.; Saidelles, F. L. F.; Kemerich, P. D. C.; Steffen, R. B.; Swarowsky, A. Silva, A. S. Crescimento e qualidade de mudas de timbó e dendaleiro cultivadas em solo contaminado por cobre. Revista Brasileira de Engenharia Agrícola e Ambiental, v.16, p.881-886, 2012.

Silva, R. F. S.; Saidelles, F. L. F.; Silva, A. S.; Bolzan, J. S.; Influência da contaminação do solo por cobre no crescimento e qualidade de mudas de açoita-cavalo (Luehea divaricata Mart. Zucc.) e aroeira-vermelha (Schinus therebinthifolius Raddi). Ciência Florestal, v.21, p.111-118, 2011.
Smith, S. E.; Read, D. J. Mycorrhizal symbiosis. 3.ed., San Diego: Academic Press, 2008. 787p.

Souza, E. L.; Antoniolli, Z. I.; Machado, R. G.; Eckardht, D. P. ; Dahmer, S. F. B.; Schirmer, G. K. Efeito da inoculação com isolados de fungos ectomicorrízicos sobre o desenvolvimento de mudas de Eucalyptus grandis Hill ex Maiden. Ciência Florestal, v.22, p.251-261, 2012.

Steffen, R. B.; Antoniolli, Z. I.; Steffen, G. P. K. Efeito estimulante do óleo essencial de eucalipto na germinação e no crescimento inicial de mudas de Eucalyptus grandis. Pesquisa Florestal Brasileira, v.30, p.199-206, 2010.

Steffen, R. B.; Antoniolli, Z. I.; Stefen, G. P. K.; Jacques, R. J. S.; Silva, R. F. S. Ação do óleo essencial de eucalipto na micorrização e no estabelecimento de mudas de Eucalyptus grandis em solo contaminado por cobre. Pesquisa Florestal Brasileira, v.31, p.245-255, 2011.

Steffen, R. B.; Antoniolli, Z. I.; Stefen, G. P. K.; Silva, R. F. Óleo essencial de Eucalyptus grandis Hill ex Maiden no estímulo à micorrização de mudas de sibipiruna (Caesalpinia peltophoroides Benth.). Ciência Florestal, v.22, p.69-78, 2012.

Taiz, L.; Zeiger, E. Fisiologia vegetal. 5.ed, Porto Alegre: Artmed, 2012. 954p.

Vitti, A. M. S.; Brito, J. O. Óleo essencial de eucalipto. São Paulo: ESALQ/USP, 2003. 26p.

Wenke, K.; Kai, M.; Piethulla, B. Belowgrond volatiles facilitate interactions between plant roots and soil organisms. Planta, v.231, p.499-506, 2010. 